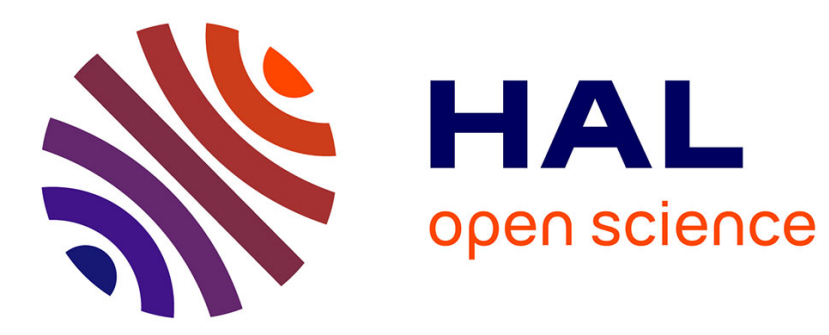

\title{
Influence of boundary conditions on the existence and stability of minimal surfaces of revolution made of soap films
}

Louis Salkin, Alexandre Schmit, Pascal Panizza, Laurent Courbin

\section{To cite this version:}

Louis Salkin, Alexandre Schmit, Pascal Panizza, Laurent Courbin. Influence of boundary conditions on the existence and stability of minimal surfaces of revolution made of soap films. American Journal of Physics, 2014, 82, pp.839. 10.1119/1.4879541 . hal-01120475

\section{HAL Id: hal-01120475 \\ https://hal.science/hal-01120475}

Submitted on 25 Feb 2015

HAL is a multi-disciplinary open access archive for the deposit and dissemination of scientific research documents, whether they are published or not. The documents may come from teaching and research institutions in France or abroad, or from public or private research centers.
L'archive ouverte pluridisciplinaire $\mathbf{H A L}$, est destinée au dépôt et à la diffusion de documents scientifiques de niveau recherche, publiés ou non, émanant des établissements d'enseignement et de recherche français ou étrangers, des laboratoires publics ou privés. 


\title{
Influence of boundary conditions on the existence and stability of minimal surfaces of revolution made of soap films
}

\author{
Louis Salkin, Alexandre Schmit, Pascal Panizza, ${ }^{*}$ and Laurent Courbin ${ }^{\dagger}$ \\ IPR, UMR CNRS 6251, Campus Beaulieu, Université Rennes 1,35042 Rennes, France \\ (Received; Accepted)
}

\begin{abstract}
Because of surface tension, soap films seek the shape that minimizes their surface energy, and thus their surface area. This mathematical postulate allows one to predict the existence and stability of simple minimal surfaces. After briefly recalling classical results obtained in the case of symmetric catenoids that span two circular rings with the same radius, we discuss the role of boundary conditions on such shapes, working with two rings having different radii. We then investigate the conditions of existence and stability of other shapes that include two portions of catenoids connected by a planar soap film and half-symmetric catenoids for which we introduce a method of observation. We report a variety of experimental results including metastability - an hysteretic evolution of the shape taken by a soap film - explained using simple physical arguments. Working by analogy with the theory of phase transitions we conclude by discussing universal behaviors of the studied minimal surfaces in the vicinity of their existence thresholds.
\end{abstract}

\section{INTRODUCTION}

Minimal surfaces have been extensively studied for centuries by both physicists and mathematicians. ${ }^{1,2}$ Applications include optimization problems such as the Steiner tree $^{2,3}$ crystallography ${ }^{4}$, and even architecture. ${ }^{5,6}$ Despite the wide literature on the topic, minimal surfaces still raise challenging questions in physics ${ }^{7}$ and in mathematics. ${ }^{8,9}$ These surfaces can be easily materialized at low cost by withdrawing wire frameworks from a soap solution to create a soap film in the frame with a (locally) minimum surface area because of surface tension. These soap-film systems can exhibit surprising behaviors bearing analogies with phase transitions including, e.g., metastability. ${ }^{10-13}$ For these reasons, minimal surfaces and soap films in particular are topics of value to education that can help the learning of theoretical and experimental physics. ${ }^{14}$ Dynamical aspects of minimal surfaces are also documented in the literature, for instance the collapse of soap films or smectic films ${ }^{15-18}$ or the deformation of a soap film induced by an impacting projectile in the context of foam physics. ${ }^{19}$ Here, we focus on the static description of minimal surfaces of revolution made of soap films.

We build on Euler's original work ${ }^{20}$ to investigate the influence of boundary conditions on the existence and stability of such surfaces. We provide generalized ways to derive familiar results and we draw connections between soap films and the physics of phase transitions. Our paper is organized as follows. In Sec. II, we begin by treating the case of a catenoid formed between two circular rings having either identical or different radii. We then study the shape adopted by a soap film when withdrawing a single ring from a bath containing a soap solution. In Sec. III, we investigate the existence and stability of a soap-film structure consisting of two portions of catenoids connected by a planar film. Finally, in Sec. IV we use arguments borrowed from the theory of phase transitions to discuss the universal nature of these minimal surfaces close to their existence thresholds.

\section{CATENOIDS}

A catenoid is a minimal surface that spans two coaxial circular rings. ${ }^{20}$ As derived in the Appendix and shown in Fig. 1, a catenoid is a surface of revolution generated from a catenary curve having a radius

$$
\rho(z)=a \cosh \left(\frac{z}{a}+C\right),
$$

where $a$ and $C$ are two parameters set by the boundary conditions imposed by the two rings. We next discuss the existence and stability of catenoids considering both symmetric $\left(R_{1}=R_{2}=R\right)$ and asymmetric $\left(R_{2}>R_{1}\right)$ cases, $R_{1}$ and $R_{2}$ being the radii of the rings (see Fig. 1 ).

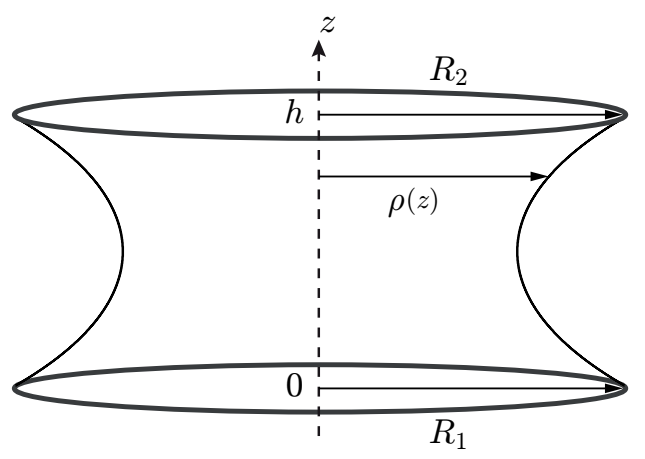

FIG. 1. Sketch of a catenoid formed between two circular rings of radii $R_{1}$ and $R_{2}$, separated by a distance $h$. The axisymmetric shape is described by $\rho(z)$.

\section{A. The symmetric catenoid}

We begin by studying the case $R_{1}=R_{2}=R$. The two boundaries conditions $\rho(0)=\rho(h)=R$ imposed by the presence of the circular rings at $z=0$ and $z=h$ give $C=-h / 2 a$ and $a \cosh (h / 2 a)=R$. This latter 
relationship can be written in the dimensionless form

$$
f(X, \Delta)=\cosh \left(\frac{\Delta X}{2}\right)-X=0,
$$

where $X=R / a$ and $\Delta=h / R$. For a given value of $\Delta$, this implicit equation can be solved graphically by plotting $f(X, \Delta)$ as a function of $X$ (Fig. 2). Because of the symmetry of the shape, the neck of the catenoid $a$ - the minimum value of $\rho(z)$-is located at $z=h / 2$. As shown in Fig. 2, three cases can be distinguished depending on the value of $\Delta$ :

- $\Delta<\Delta_{c} \approx 1.33$. For a given value of $\Delta$, Eq. (2) admits two roots for $X$. Two different catenoids can therefore exist between two rings having the same radius. One can easily show that the catenoid having the largest neck has the lowest energy.

- $\Delta=\Delta_{c}$. Here one finds a single root $\left(X_{c} \simeq 1.81\right)$ so that only one catenoid satisfies the given boundary conditions.

- $\Delta>\Delta_{c}$. In this regime Eq. (2) has no solution because $f(X, \Delta)>0$ for any $X$ (see Fig. 2). Hence, no catenoid can exist between the two rings for this range of $\Delta$.

The critical point corresponding to the loss of existence of solutions can be analytically determined by solving the set of equations $f\left(X_{c}, \Delta_{c}\right)=0$ and $\partial_{X} f\left(X_{c}, \Delta_{c}\right)=0$, which gives the implicit equation

$$
\operatorname{coth}\left(\frac{\Delta_{c} X_{c}}{2}\right)=\frac{\Delta_{c} X_{c}}{2} .
$$

Equation (3) has the unique solution $\Delta_{c} X_{c} / 2 \approx 1.20$. By using this result and $f\left(X_{c}, \Delta_{c}\right)=0$, one can obtain expressions for the existence thresholds $a_{c} / R=1 / X_{c} \approx$ 0.55 and $h_{c} / R=\Delta_{c} \approx 1.33$.

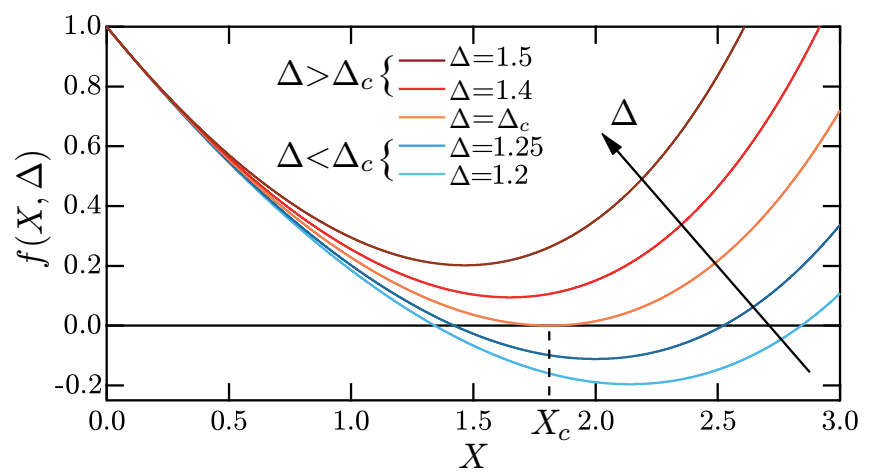

FIG. 2. (Color online) Solution of Eq. (2) imposed by the two boundary conditions $\rho(0)=\rho(h)=R$ in the case of a symmetric catenoid.

We next validate experimentally these well-known predictions. In all of our experiments except those of
Sec. II C, we use a digital caliper with two circular coaxial rings (radius $R=0.5-5 \mathrm{~cm}$ ) made of wire $(1 \mathrm{~mm}$ in diameter) attached to the caliper's jaws; care is taken to ensure that the rings are parallel. This device enables us to measure the distance $h$ precisely, the neck radius $a$ being determined by imaging the shape of the soap film with a camera (EO-1312C, Edmund Optics). We create symmetric catenoids by withdrawing two identical rings perpendicularly from a liquid bath containing a soap solution. This solution is made of water ( $80 \%$ by volume), commercial dishwashing liquid (Fairy, Procter \& Gamble, $7 \%$ by volume), and glycerol ( $13 \%$ by volume). The air-solution surface tension $\gamma=25 \mathrm{mN} / \mathrm{m}$ is measured using pendant drop tensiometry (Tracker, Teclis).

After removal of the frame, a so-called diaboloid surface (discussed in more detail in Sec. III) is first observed between the rings. This shape consists of two catenoids connected to each other by a planar soap film parallel to the rings. We begin an experiment by bursting the planar film so that the shape relaxes to become a symmetric catenoid that we study as a function of the distance $h$. Figure 3 shows the measurements of the normalized neck radius $a / R=1 / X$ as a function of $\Delta$. Although two catenoid solutions can be found mathematically when $\Delta<\Delta_{c}$, only the catenoid having the larger neck (and lower surface energy) is observed experimentally. When $\Delta=\Delta_{c}$, the catenoid collapses into two planar films suspended on the two rings. This type of behavior bears strong analogies with bifurcation diagrams classically observed in the physics of instabilities.
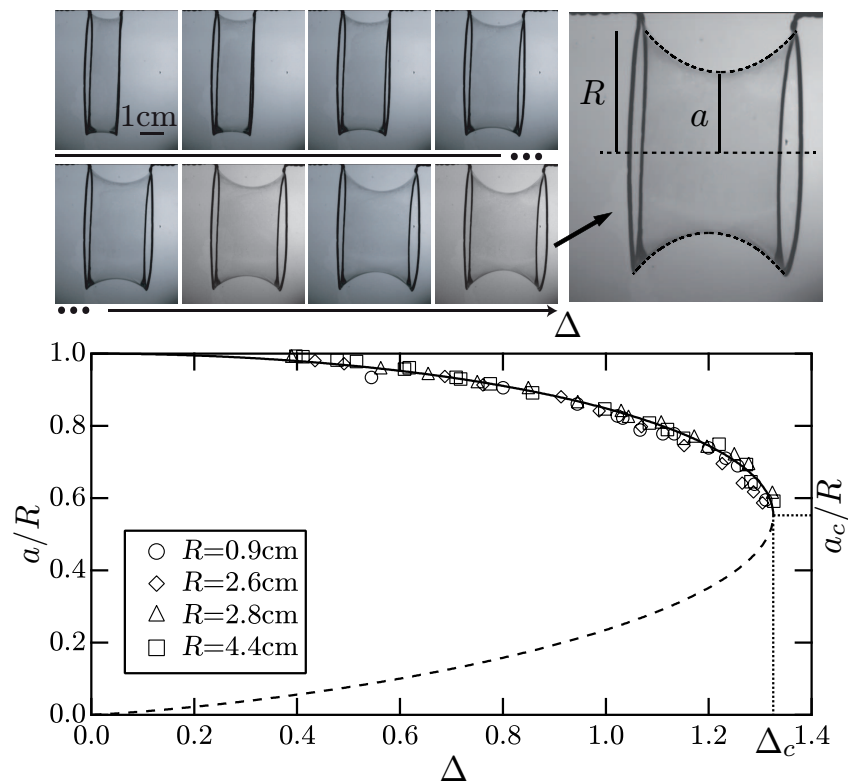

FIG. 3. Top panel: photographs showing the evolution of a symmetric catenoid when $\Delta=h / R$ increases. Bottom panel: experimental evolution of the normalized neck radius $a / R$ as a function of $\Delta$. Each symbol stands for a given value of $R$ as indicated. The solid and dashed curves depict the stable and unstable theoretical solutions, respectively (the stable one corresponding to the catenoid having the largest neck). 


\section{B. The asymmetric catenoid}

To study the influence of the boundary conditions on the existence and stability of a catenoid, we now work with rings having different radii $R_{1}$ and $R_{2}=\alpha R_{1}$, with $\alpha>1$; as previously (see Fig. 1 ) the two rings are located at $z=0$ and $z=h$, respectively. The boundary conditions now read

$$
\begin{gathered}
\rho(0)=a \cosh C=R_{1}, \\
\rho(h)=a \cosh \left(\frac{h}{a}+C\right)=R_{2} .
\end{gathered}
$$

Using the relation $\cosh C=X=R_{1} / a$ and trigonometric identities, Eq. (4b) can be written in the dimensionless form

$$
f_{\alpha}(X, \Delta)=\sinh ^{2}(\Delta X)-X^{2}\left[2 \alpha \cosh (\Delta X)-1-\alpha^{2}\right]=0,
$$

where $\Delta=h / R_{1}$.

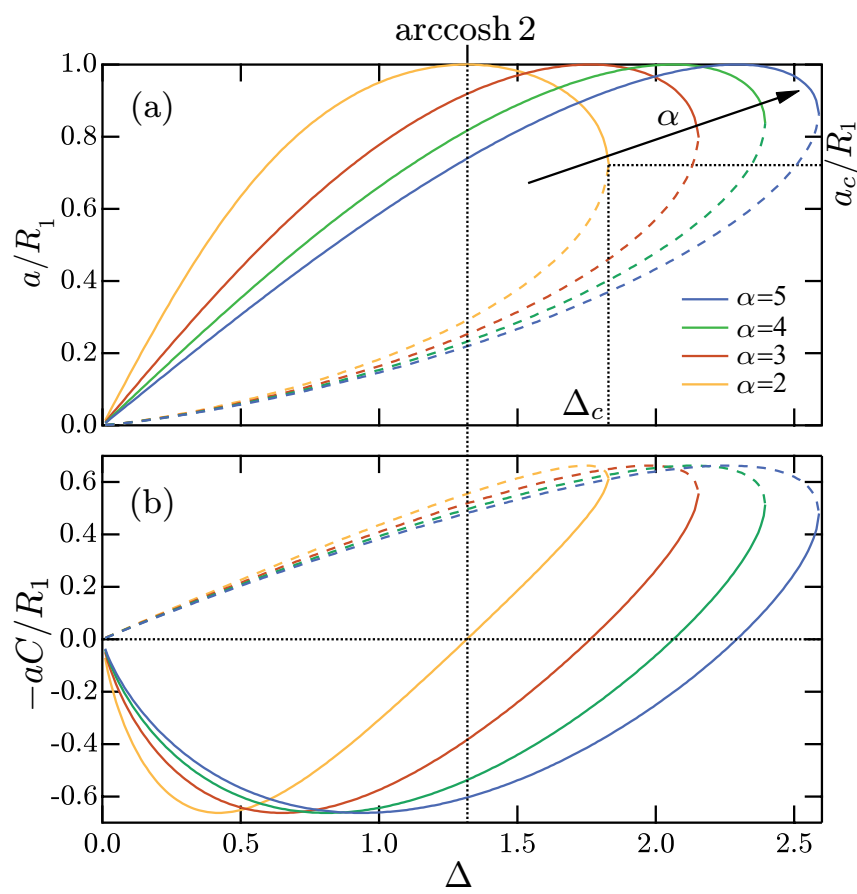

FIG. 4. (Color online) Theoretical evolution of (a) $a / R_{1}$ and (b) the normalized position of the neck radius $-a C / R_{1}$ as a function of $\Delta=h / R_{1}$. The solid and dashed curves correspond to the stable and unstable solutions, respectively. Each shade of gray (color online) represents a given value of $\alpha$ as indicated. The coordinates of the point at which the value $a / R_{1}$ of a given stable branch is maximum are $(\operatorname{arccosh} \alpha, 1)$, as discussed in the text. When $\Delta=\operatorname{arccosh} \alpha$, the neck radius is located at $z=0$.

Solving Eq. (5), one obtains the evolution of $a / R_{1}$ as a function of $\Delta$ for different values of $\alpha$ (see Fig. 4). Comparing the symmetric ( $\alpha=1$ in Fig. 3 ) and asymmetric $(\alpha>1$ in Fig. 4) cases we observe some similarities in the response. For any $\alpha>1$, when $\Delta$ is lower than a critical value $\Delta_{c}$, two solutions are found for Eq. (5) so that two asymmetric catenoids can exist, the one with the larger neck having the smaller surface energy. Above $\Delta_{c}$, Eq. (5) has no solutions so no catenoid can exist.

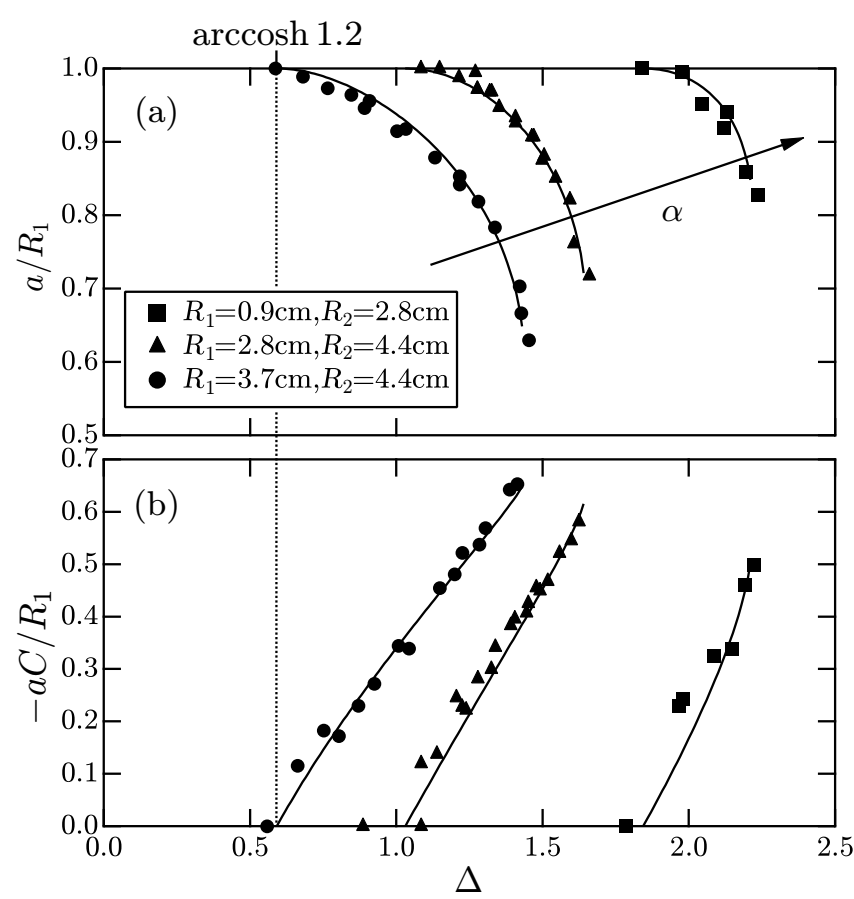

FIG. 5. Experimental evolution of (a) $a / R_{1}$ and (b) $-a C / R_{1}$ as a function of $\Delta=h / R_{1}$, with $\alpha=1.2(\bullet), 1.6(\boldsymbol{\Delta}), 3.1(\boldsymbol{\square})$. The solid curves correspond to theoretical predictions for the stable solutions. As indicated in the figure and explained in the text, measurements can be carried out only when $\Delta \geq$ $\operatorname{arccosh} \alpha$.

In contrast with the symmetric case, when $\Delta<\Delta_{c}$, the variations of $a / R_{1}$ with $\Delta$ for the stable solutions are nonmonotonic [solid lines in Fig. 4 (a)]. In addition, the position of the neck radius $z=-a C$ is no longer located at the midpoint between the two rings as shown by the variations of $-a C / R_{1}$ with $\Delta$ [see Fig. 4 (b)]. Indeed, our results for the stable solutions show that $C$ is positive when $a$ increases with $\Delta$ and its sign becomes negative for large $\Delta$ when $a$ decreases with this quantity. Consequently, the position of the neck radius is located between the two rings only for large enough $\Delta$ when $C$ is negative. The neck radius is at $z=0$ when $C=0$ and by using Eq. (4) one finds that this occurs when $\Delta=\operatorname{arccosh} \alpha$ (see Fig. 4). To summarize, one predicts that the neck radius of a catenoid is experimentally measurable only when $\Delta \geq \operatorname{arccosh} \alpha$ and it is located at $z=0$ (i.e. $a=R_{1}$ ) when $\Delta=\operatorname{arccosh} \alpha$ (note that for the symmetric case $\operatorname{arccosh} 1=0)$. We use the soap solution described in Sec. II A to validate these predictions experimentally. As shown in Fig. 5, the resulting variations of $a / R_{1}$ and $-a C / R_{1}$ with $\Delta$, measured for different values of $\alpha$, show good agreement with the predictions. 
Following a procedure similar to the one employed in Sec. II A, the existence thresholds $a_{c} / R_{1}=1 / X_{c}$ and $\Delta_{c}$ can be determined by solving $f_{\alpha}\left(X_{c}, \Delta_{c}\right)=0$ and $\partial_{X} f_{\alpha}\left(X_{c}, \Delta_{c}\right)=0$. Although these two equations are nonlinear and coupled, they can be solved using a numerical method as follows. By combining these two equations, one finds the following implicit relation that admits $\Delta_{c} X_{c}$ as a root and that can be solved numerically:

$$
\begin{gathered}
\tanh \left(\Delta_{c} X_{c}\right)=\Delta_{c} X_{c}\left(2 \alpha \cosh \left(\Delta_{c} X_{c}\right)-1-\alpha^{2}\right) / \\
{\left[\alpha\left(2 \cosh \left(\Delta_{c} X_{c}\right)+\Delta_{c} X_{c} \sinh \left(\Delta_{c} X_{c}\right)\right)-1-\alpha^{2}\right] .}
\end{gathered}
$$

Using the result of the resolution of Eq. (6) to solve $f_{\alpha}\left(X_{c}, \Delta_{c}\right)=0$, one obtains the numerical evolution of $X_{c}$ and subsequently the variations of $\Delta_{c}$ as a function of $\alpha$. Although we use a slightly different approach, our numerical method is in full agreement with other geometric demonstrations published in the literature. ${ }^{21}$ As shown in Fig. 6, the existence thresholds are monotonically increasing functions of $\alpha$, which indicates that the asymmetry increases the domain of existence of catenoids. Our numerical predictions agree reasonably well with the experimental results.

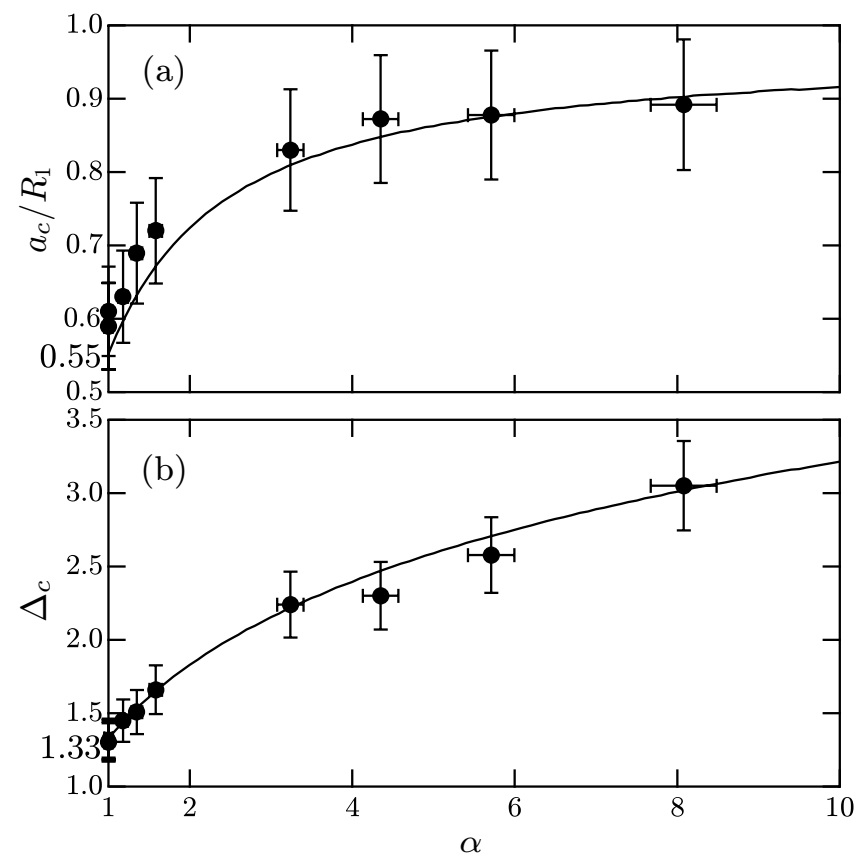

FIG. 6. Existence thresholds (a) $a_{c} / R_{1}$ and (b) $\Delta_{c}$ of asymmetric catenoids as a function of $\alpha$ characterizing the asymmetry: comparison between predictions (solid curves) and experiments (data points).

\section{The half-symmetric catenoid}

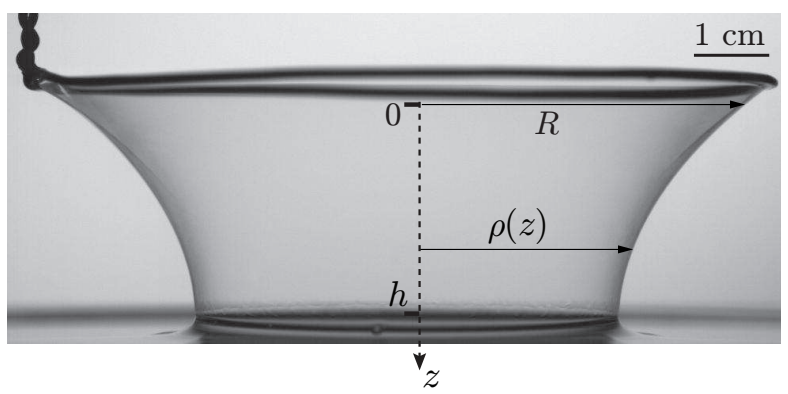

FIG. 7. Image showing the soap film formed when withdrawing a ring from a bath of soap solution and showing the parameters $R, h$, and $\rho(z)$.

Withdrawing a circular ring of radius $R$ at a height $h$ from a bath of soap solution creates an axisymmetric soap film as shown in Fig. 7. Above a critical height $h_{c}$, this shape collapses to leave a planar film on the ring and a soap bubble in contact with the bath. ${ }^{22}$ Similar to the shapes studied in previous sections, this surface of revolution is also a catenoid. The origin of this shape resides in its specific boundary conditions. Indeed, in this case only a single boundary condition, $\rho(0)=R$, is imposed by the operator. When $h<h_{c}$, experiments show that the radius of the soap film at the level of the bath, $\rho(h)$, is a decreasing function of $h$. In addition, careful observations at $z=h$ reveal that the soap film is perpendicular to the horizontal surface of the bath for any values of $R$ and $h$. This result - a direct consequence of the mechanical balance of the forces acting on the liquid meniscus connecting the bath and the soap film-gives rise to the second boundary condition: $\rho^{\prime}(h)=0$. Hence, using Eq. (1), the boundary conditions read

$$
\begin{gathered}
\rho(0)=a \cosh C=R, \\
\rho^{\prime}(h)=\sinh \left(\frac{h}{a}+C\right)=0 .
\end{gathered}
$$

As seen in other problems in physics, when we compare the case of the symmetric catenoid with that of the present shape, it is worth noting that the change in boundary conditions at $z=h$ corresponds to a transition from Dirichlet $[\rho(0)=\rho(h)=R]$ to mixed DirichletNeumann $\left[\rho(0)=R, \rho^{\prime}(h)=0\right]$ conditions.

Using Eqs. (7), one finds $C=-h / a$ and $a \cosh (h / a)=$ $R$. Apart from the factor of $1 / 2$, these results are strictly analogous to those obtained for symmetric catenoids (see Sec. II A). We therefore interpret the shape in Fig. 7 as half of a symmetric catenoid whose neck radius $\rho(h)=a$ is located at the level of the bath. Thus, existence thresholds can be guessed using previous results (Sec. II A): $1 / X_{c}=a_{c} / R \approx 0.55, \Delta_{c}=h_{c} / R \approx 0.66$. As shown in 

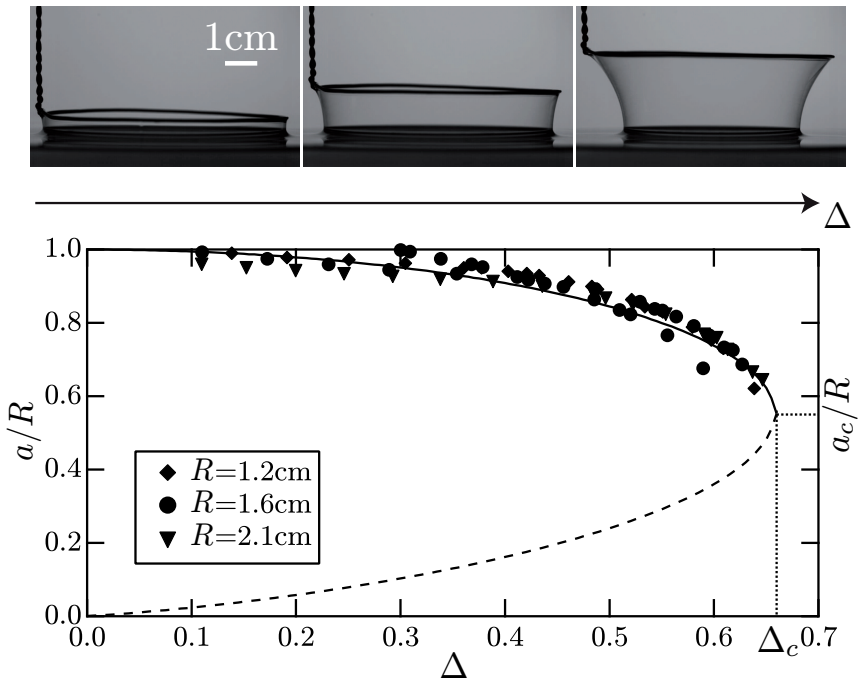

FIG. 8. Top panel: photographs of a typical experiment showing the evolution of a half-symmetric catenoid as $\Delta=h / R$ increases. Bottom panel: experimental evolution of the normalized neck radius $\frac{a}{R}$ as a function of $\Delta$. The solid and dashed curves stand for the stable and unstable theoretical solutions, respectively.

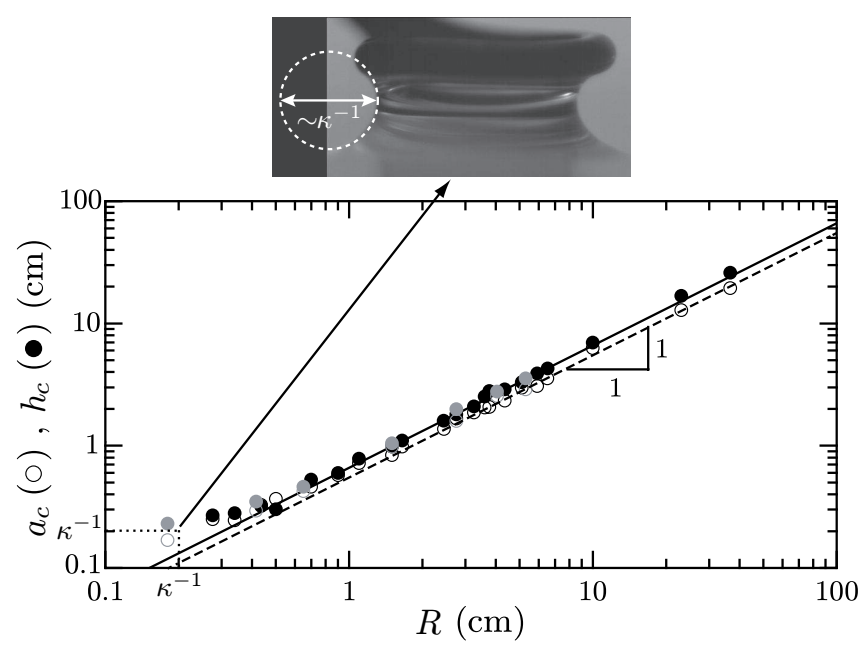

FIG. 9. Top panel: typical liquid meniscus observed when $\Delta<\Delta_{c}$ with a ring of radius $R \sim \kappa^{-1}, \kappa^{-1}$ being the capillary length. Bottom panel: predicted thresholds $a_{c} \approx 0.55 R$ (dashed line) and $h_{c} \approx 0.66 R$ (solid line) as a function of $R$. The black and gray symbols correspond, respectively, to measurements made with the soapy solution described in Sec. II A and the Sodium Docecyl Sulfate solution.

Fig. 8), experimental results agree well with these predictions.

We also conduct similar experiments with a mixture of water and a surfactant (Sodium Dodecyl Sulfate) at a concentration of $15 \mathrm{~g} / \mathrm{L}$. For both this mixture and the soap solution described in Sec. II A, our predictions capture the experimental thresholds as long as $R$ is larger than a couple of millimeters (see Fig. 9). We attribute the deviation observed at small $R$ to capillary effects related to the presence of the liquid meniscus at the level of the bath not taken into account in our simple model. The typical size of this meniscus is given by the capillary length ${ }^{23} \kappa^{-1}=\sqrt{\gamma / \rho g} \approx 2 \mathrm{~mm}$, with $\rho$ the liquid density, $\gamma$ the air-liquid surface tension, and $g$ the gravitational field strength. When $R \lesssim \kappa^{-1}$, meniscus effects come into play and set the thresholds; a more detailed description necessary in this case is beyond the scope of this paper.

\section{DIABOLOIDS}

We now study the existence and stability of shapes that span two rings of radii $R_{1}$ and $R_{2}$ and are composed of two portions of catenoids connected to each other by a planar film (see Fig. 10). In what follows, we refer to these surfaces as "diaboloids" because they resemble a well-known juggling object, the diabolo. ${ }^{24}$

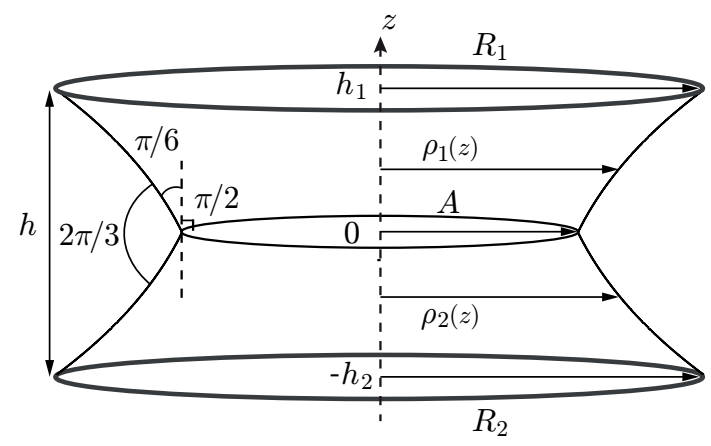

FIG. 10. Sketch of a diaboloid defining the variables $R_{1}, R_{2}$, $h, h_{1}, h_{2}, A, \rho_{1}(z)$, and $\rho_{2}(z)$.

Such minimal surfaces have been used as model systems $^{7,25-27}$ to address experimental problems in the context of the physics of foams. Here, our goal is to study how the presence of the planar soap film affects the existence and stability of this surface with respect to those of the associated catenoid that would form between two similar rings as discussed in Sec. II.

\section{A. The symmetric diaboloid}

We begin by considering the symmetric case, for which $R_{1}=R_{2}=R$. The profiles of the upper and lower catenoids are given by (see Fig. 10)

$$
\begin{aligned}
& \rho_{1}(z)=a_{1} \cosh \left(\frac{z}{a_{1}}+C_{1}\right), \\
& \rho_{2}(z)=a_{2} \cosh \left(\frac{z}{a_{2}}+C_{2}\right) .
\end{aligned}
$$


The boundary conditions determined at $z=0, z=h_{1}$, and $z=-h_{2}$ yield

$$
\begin{gathered}
A=a_{1} \cosh C_{1}=a_{2} \cosh C_{2}, \\
R_{1}=a_{1} \cosh \left(\frac{h_{1}}{a_{1}}+C_{1}\right), \\
R_{2}=a_{2} \cosh \left(\frac{-h_{2}}{a_{2}}+C_{2}\right), \\
h=h_{1}+h_{2} .
\end{gathered}
$$

This system of five equations does not enable one to determine the seven unknown parameters $\left(a_{1}, C_{1}, h_{1}, a_{2}, C_{2}, h_{2}, A\right)$; two supplementary equations are necessary for the system not to be underdetermined. The needed equations are given by the mechanical balance of the forces acting on the Plateau border between the planar film and the two portions of catenoids (Fig. 10). In the dry limit, ${ }^{25}$ the third Plateau's law imposes that the three angles made by the films connected to the Plateau border are all equal to $2 \pi / 3 .^{1,28}$ Mathematically, this can be written as

$$
\begin{gathered}
\rho_{1}^{\prime}(z=0)=\sinh C_{1}=\tan \left(\frac{\pi}{6}\right), \\
\rho_{2}^{\prime}(z=0)=\sinh C_{2}=-\tan \left(\frac{\pi}{6}\right) .
\end{gathered}
$$

As a result, $C_{1}=-C_{2}=C \approx 0.55$ and $a_{1}=a_{2}=$ $a=A / \cosh C$. It is worth noting that the profile of the associated catenoid, for which the slope is zero at the midpoint between rings, can be retrieved by writing $\rho^{\prime}(z=0)=\sinh C=0$ so that $C=0$. The apparent discrepancy with the result discussed in Sec. II A $(C=$ $-h / 2 a)$ stems from the different location taken for the origin of the $z$-axis. Using these results and Eq. (9) we find

$$
h_{1}=h_{2}=\frac{h}{2}=a\left[\operatorname{arccosh}\left(\frac{R}{a}\right)-C\right] .
$$

Since the radius of the planar soap film $A$ is easily accessible experimentally when compared to $a$, we next derive the existence thresholds of the diaboloid in terms of the dimensionless quantities $A / R$ and $h / R$. The boundary condition at $z=h_{1}$ can be expressed in the (dimensionless) form

$$
g(X, \Delta)=\cosh \left(\frac{\Delta X}{2}+C\right)-X=0
$$

with $X=R / a$ and $\Delta=h / R$. This implicit equation admits two roots when $\Delta$ is smaller than a critical value $\Delta_{c}$. When $C=0$, we retrieve the equation derived in the case of a symmetric catenoid [see Eq. (2)]. In fact, the resolution of Eq. (12) is qualitatively analogous to that of Eq. (2). The existence thresholds for the symmetric diaboloid are obtained by solving $g\left(X_{c}, \Delta_{c}\right)=0$ and $\partial_{X} g\left(X_{c}, \Delta_{c}\right)=0$.

Following the approach employed in Sec. II, we find that $\Delta_{c} X_{c} / 2 \approx 1.08$ is the solution of the implicit equation

$$
\operatorname{coth}\left(\frac{\Delta_{c} X_{c}}{2}+C\right)=\frac{\Delta_{c} X_{c}}{2}
$$

Using this result and solving $g\left(X_{c}, \Delta_{c}\right)=0$ we obtain $A_{c} / R=\cosh C / X_{c} \approx 0.44$ and $h_{c} / R=\Delta_{c} \approx 0.82$. The value of $\Delta_{c}$ was inferred by other authors based on experimental data. ${ }^{25}$ We note that the critical distance $h_{c}$ of a diabloid is relatively smaller than that of the associated catenoid for which $\Delta_{c} \approx 1.33$. This result suggests that the third Plateau's law is an additional constraint for diaboloids structures that makes them less stable than catenoids. As shown in Fig. 11, experiments mirror our theoretical predictions.
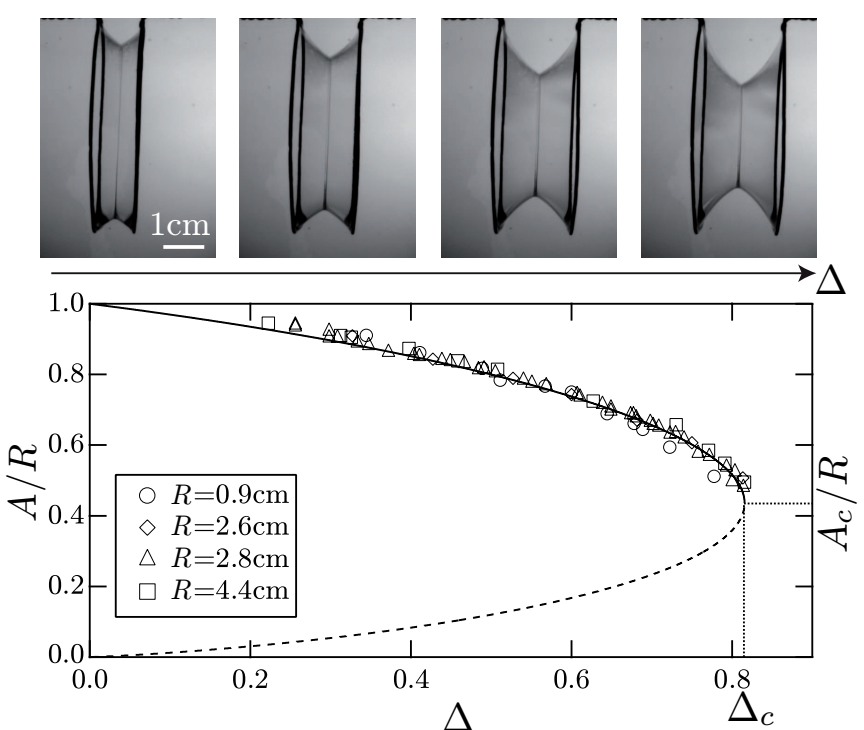

FIG. 11. Top panel: photographs of a typical experiment showing the evolution of a symmetric diaboloid as $\Delta$ increases. Bottom panel: experimental variations of the normalized radius of the planar film $A / R$ as a function of the normalized distance $\Delta=h / R$. The symbols stand for different values of $R$ as indicated. The solid and dashed curves correspond to the stable and unstable theoretical solutions, respectively.

\section{B. The asymmetric diaboloid}

To investigate the asymmetric case, we again consider two different rings with radii $R_{1}$ and $R_{2}=\alpha R_{1}$ with $\alpha>$ 1. This symmetry breaking yields significant changes in the behavior of the diaboloid. The boundary conditions at $z=h_{1}$ and $z=-h_{2}$ [see Eqs. (9b) and (9c) and 
Fig. 10] yield

$$
\begin{gathered}
\cosh \left(\Delta_{1} X+C\right)=X, \\
\cosh \left(\Delta_{2} X+C\right)=\alpha X,
\end{gathered}
$$

where $X=R_{1} / a$ and $\Delta_{i}=h_{i} / R_{1}$ with $i=1$ or 2 . Combining Eqs. (14a) and (14b) we find

$$
g_{\alpha}(X, \Delta)=\operatorname{arccosh} X+\operatorname{arccosh}(\alpha X)-\Delta X-2 C=0 .
$$

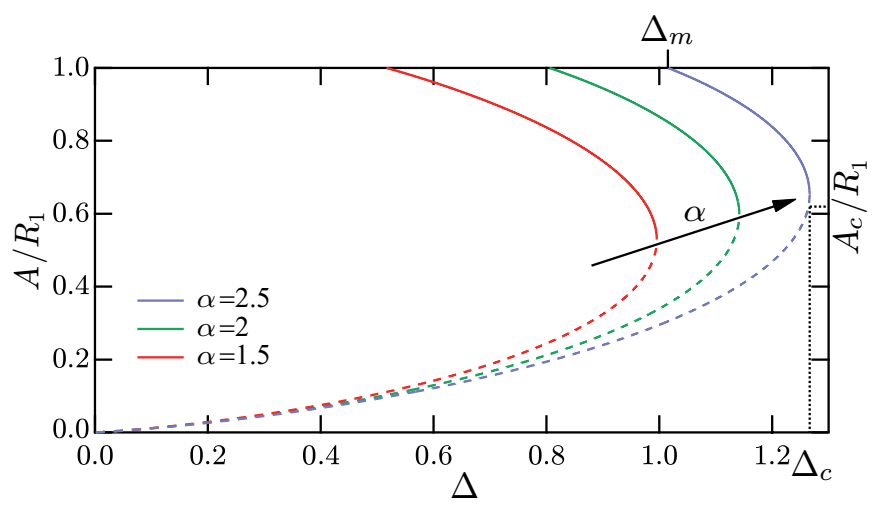

FIG. 12. (Color online) Theoretical evolution of the normalized radius of the planar soap film $A / R_{1}$ as a function of the normalized distance $\Delta=h / R_{1}$. The solid and dashed curves correspond to the stable and unstable solutions, respectively. Different shades of gray correspond to different values of $\alpha$, as indicated. The coordinates of $\Delta_{m}$ are $([\operatorname{arccosh}(\alpha \cosh C)-C] / \cosh C, 1)$, as discussed in the text.

Figure 12 shows the evolution of $A / R_{1}$ as a function of $\Delta=h / R_{1}$ for different values of $\alpha$. As previously shown in the case of asymmetric catenoids, the existence thresholds $\Delta_{c}$ and $A_{c} / R_{1}$ are increasing functions of $\alpha$. Surprisingly, when comparing the symmetric (Fig. 11) and asymmetric (Fig. 12) cases, stable asymmetric diaboloids have smaller domains of existence than symmetric ones. Indeed, Fig. 12 shows that an asymmetric diaboloid exists only when $\Delta_{m} \leq \Delta \leq \Delta_{c}$ for any $\alpha$. An expression for $\Delta_{m}$ can be derived by having $A$ take its maximal value $\left(A=R_{1}\right)$. When $\Delta<\Delta_{m}$, writing Eq. (9b) with $R_{1}=A$ in this case of asymmetric shapes, one finds that $h_{1}=0$, i.e. the most stable minimal surface is composed of an asymmetric catenoid built on the two rings and a planar soap film suspended on the smaller ring. The resolution of the boundary conditions [Eq. (9)] with $A=R_{1}$ yields

$$
\Delta_{m}=\frac{\operatorname{arccosh}(\alpha \cosh C)-C}{\cosh C},
$$

with $C \approx 0.55$. It is straightforward to show that $\Delta_{m}=0$ in the symmetric case $(\alpha=1)$.

Similar to the previously discussed cases, the existence thresholds $X_{c}$ and $\Delta_{c}$ are determined by solving $g_{\alpha}\left(X_{c}, \Delta_{c}\right)=0$ and $\partial_{X} g_{\alpha}\left(X_{c}, \Delta_{c}\right)=0$, which lead to

$$
\operatorname{arccosh} X_{c}+\operatorname{arccosh}\left(\alpha X_{c}\right)=\Delta_{c} X_{c}+2 C,
$$

$$
\frac{1}{\sqrt{X_{c}^{2}-1}}+\frac{\alpha}{\sqrt{\left(\alpha X_{c}\right)^{2}-1}}=\Delta_{c} .
$$

We confront our theoretical predictions with experimental variations of $A / R_{1}$ with $\Delta$ (see Fig. 13). Interestingly, our experiments reveal the existence of an hysteretic behavior, a phenomenon that is a typical signature of a subcritical bifurcation in the physics of instabilities. Indeed, experimental data differ depending on whether the ring separation distance rings $h$ is continuously increased or decreased (Fig. 13). This hysteresis cycle is an illustration of a phenomenon of metastability resulting from the coexistence of two minimal surfaces: the asymmetric diaboloid characterized by $A / R_{1}<1$, and the asymmetric catenoid associated with a planar soap film on the smaller ring characterized by $A / R_{1}=1$. We will refer to these two configurations as $D$ (diaboloid) and $C$ (catenoid), respectively. Also, we denote as $\Delta_{C \rightarrow D}$ the value of $\Delta$ for which the system undergoes the transition from the configuration $C$ to the configuration $D$ as $h$ increases. Similarly, the transition from the configuration $D$ to the configuration $C$ is obtained at $\Delta_{D \rightarrow C}$ when $h$ decreases (Fig. 13).

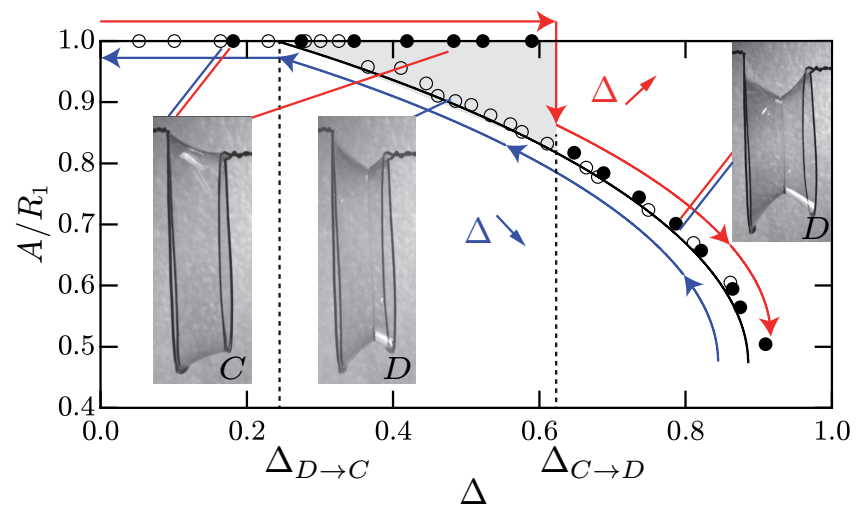

FIG. 13. (Color online) Experimental evolution of $A / R_{1}$ as a function of $\Delta=h / R_{1}$ illustrating the hysteretic response. The symbols indicate the experimental conditions: (solid) $h$ increases, (open) $h$ decreases. The experimental parameters are $R_{1}=3.7 \mathrm{~cm}, R_{2}=4.4 \mathrm{~cm}$, and $\alpha=1.2$. The solid curve corresponds to the theoretical solution of the stable diaboloid.

To rationalize the observed hysteresis and predict theoretical expressions for $\Delta_{C \rightarrow D}$ and $\Delta_{D \rightarrow C}$, we next investigate the transitions from one configuration to the other. As previously discussed, asymmetric diaboloids exist only when $\Delta_{m} \leq \Delta \leq \Delta_{c}$, hence the transition from $D$ to $C$ is observed at $\Delta_{D \rightarrow C}=\Delta_{m}=$ $[\operatorname{arccosh}(\alpha \cosh C)-C] / \cosh C$. Studying the transition from $C$ to $D$, and given that $\Delta_{C \rightarrow D} \neq \Delta_{D \rightarrow C}$, one guess is that the system is metastable in configuration $C$. Indeed, calculations (not shown here) indicate that the surface energy of state $D$, when this configuration exists, is always lower than but close to that of configuration $C$. Accordingly, we need to find a plausible transition path selected by the system that can account for the metastability. Following the classical picture used in the theory of 
chemical reactions, we propose a phenomenological twostep mechanism detailed below and depicted in Fig. 14:

(1) The planar soap film moves from the smaller ring to its final position in the configuration $D$, the profile of the asymmetric catenoid remaining unchanged.

(2) The profile of the initial asymmetric catenoid changes to accommodate the presence of the planar film by satisfying angular constraints imposed by the third Plateau's law.

This two-step mechanism suggests that the transition from $C$ to $D$ occurs only if the first step is favorable in terms of surface energy. During this step, the surface area of the planar film decreases only when the neck radius of the asymmetric catenoid is located between the two rings. Hence, using results obtained in Sec. IIB we infer that $\Delta_{C \rightarrow D}=\operatorname{arccosh} \alpha$.

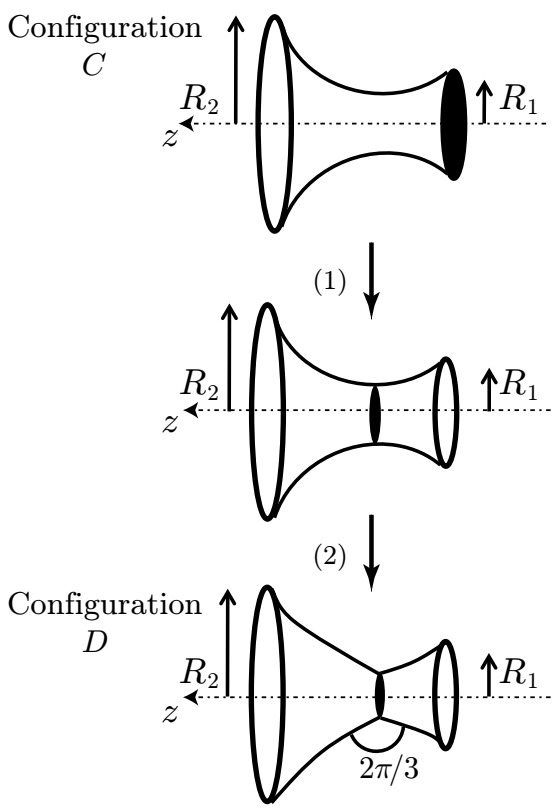

FIG. 14. Sketch of the two-step mechanism. First, the planar soap film moves without affecting the profile of the initial asymmetric catenoid. Second, the catenoid adapts its shape because of the presence of the planar film.

Figure 15 (a) shows the evolution with $\alpha$ of the variables characterizing the hysteretic behavior, $\Delta_{D \rightarrow C}$, $\Delta_{C \rightarrow D}$, and $\Delta_{c}$. As shown in this figure, our simple physical arguments help explain the experiments. Also, the predicted variations of $A / R_{1}$ with $\Delta$ for different $\alpha$ agree well with experiments [Fig. $15(\mathrm{~b})$ ]. As the predicted curves for $\Delta_{C \rightarrow D}$ and $\Delta_{c}$ intersect at $\alpha=\alpha_{c} \approx 1.56$, two different scenarios that depend on $\alpha$ are possible:

- $\alpha<\alpha_{c}, \Delta_{C \rightarrow D}<\Delta_{c}$ : when $h$ is increased one first observes the transition from $C$ to $D$ and then the collapse of the film at the existence threshold;

- $\alpha>\alpha_{c}, \Delta_{C \rightarrow D}>\Delta_{c}$ : when $h$ increases the soap film breaks at $\Delta_{C \rightarrow D}$ so that configuration $D$ is never observed. Experiments conducted for $\alpha=1.6$ correlate with this scenario [Fig. 15(b)]. To overcome this limitation and force the transition from $C$ to $D$ when $\Delta_{m}<\Delta<\Delta_{c}<\Delta_{C \rightarrow D}$, one can provide some activation energy to the system experimentally. For instance, blowing air on the soap film or touching it with a wet spatula can actively tune down the value of $\Delta_{C \rightarrow D}$ and select the configuration adopted by the film.
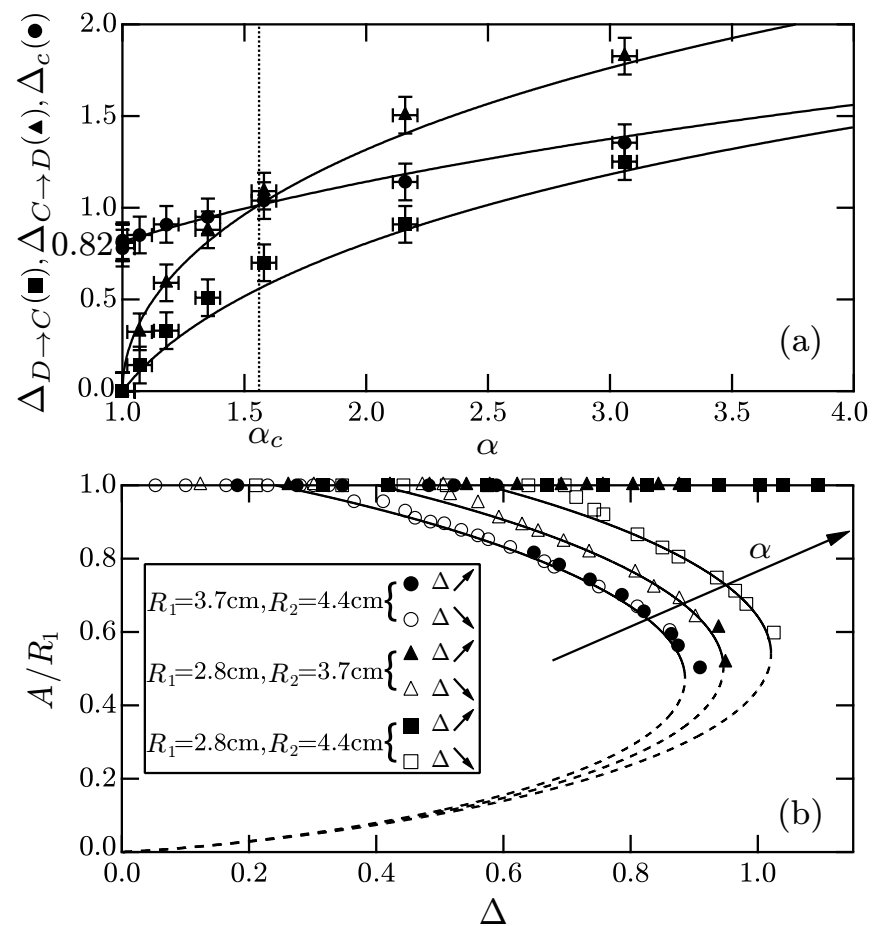

FIG. 15. (a) Variations of $\Delta_{D \rightarrow C}(\mathbf{\square}), \Delta_{C \rightarrow D}(\boldsymbol{\Lambda})$, and $\Delta_{c}(\bullet)$ as a function of $\alpha$ : comparison between experiments (points) and theory (curves). (b) Experimental evolution of $A / R_{1}$ as a function of $\Delta$. Solid and open symbols correspond to measurements taken when the rings are moved apart and closer, respectively. The shape of the symbol labels for the asymmetry: $\alpha=1.2$ (circles), 1.3 (triangles), 1.6 (squares). Solid and dashed curves correspond to the stable and unstable theoretical solutions, respectively. For the largest asymmetry $\alpha>\alpha_{c}$ (Fig. 15 (a) defines $\alpha_{c}$ ), configuration $D$ is not observed experimentally when $h$ increases.

\section{UNIVERSAL BEHAVIOR IN THE} VICINITY OF THE EXISTENCE THRESHOLD

Solving Eq. (17), one can determine the existence thresholds $\left(X_{c}, \Delta_{c}\right)$ for all minimal surfaces of revolution considered in this article. Indeed, thresholds for symmetric diaboloids and (symmetric and asymmetric) catenoids are respectively retrieved by taking $\alpha=1$ and either $C=\operatorname{arsinh}(\tan \pi / 6)$ or $C=0$ in Eq. (17). In addition, the threshold of the half-symmetric catenoid can be de- 
duced from that of the symmetric catenoid by dividing $\Delta_{c}$ by 2 . These results are summarized in Fig. 16.

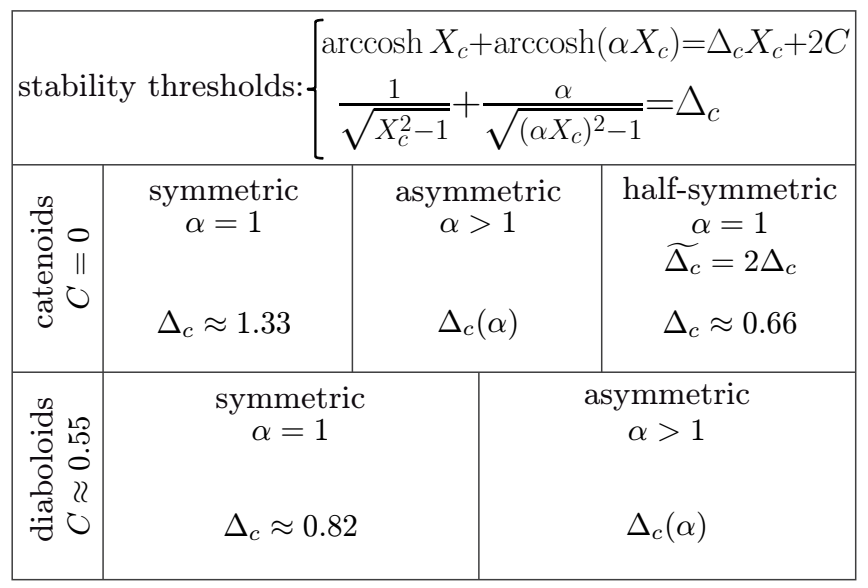

FIG. 16. Table summarizing the stability thresholds $\Delta_{c}$ derived in the text, for each minimal surface studied in this article. The thresholds are determined by Eqs. (17). In the case of half-symmetric catenoids, one determines the threshold by replacing $\Delta_{c}$ by $\widetilde{\Delta_{c}}$ in Eq. (17) when solving this set of equations.

Similar to the behavior of second-order phase transitions characterized by the same critical exponents close to their critical point, all minimal surfaces studied here present a universal behavior in the vicinity of their existence thresholds. Indeed, they are fully described by the two geometrical quantities $X$ and $\Delta$. Close to their existence thresholds, we can approximate $g_{\alpha}(X, \Delta)$ [Eq. (15)] by a second-order Taylor series. For fixed values of $C$ and $\alpha$, using $\Delta=\Delta_{c}+\delta \Delta$ and $X=X_{c}+\delta X$, we can then expand $g_{\alpha}(X, \Delta)$ as

$$
\begin{array}{r}
g_{\alpha}(X, \Delta) \simeq g_{\alpha}\left(X_{c}, \Delta_{c}\right)+\partial_{X} g_{\alpha} \delta X+\partial_{\Delta} g_{\alpha} \delta \Delta+ \\
\partial_{X X} g_{\alpha} \frac{(\delta X)^{2}}{2}+\partial_{X \Delta} g_{\alpha} \delta \Delta \delta X+\partial_{\Delta \Delta} g_{\alpha} \frac{(\delta \Delta)^{2}}{2}=0
\end{array}
$$

where all the derivatives are computed at $X=X_{c}$ and $\Delta=\Delta_{c}$. Since $X_{c}$ and $\Delta_{c}$ are solutions of $\left\{g_{\alpha}\left(X_{c}, \Delta_{c}\right)=\right.$ $\left.0, \partial_{X} g_{\alpha}\left(X_{c}, \Delta_{c}\right)=0\right\}$, by comparing the different terms of the Taylor series, we can show that

$$
\delta X \simeq \pm \sqrt{\left|\frac{2 \partial_{\Delta} g_{\alpha}}{\partial_{X X} g_{\alpha}}\right|} \sqrt{-\delta \Delta} .
$$

As $X=R / a$, we have $\delta X=-R \delta a / a_{c}^{2}$. Near the threshold, we therefore find that

$$
\frac{\delta a}{R}=\frac{a-a_{c}}{R} \simeq \pm J \sqrt{\Delta_{c}-\Delta}
$$

with

$$
J=\sqrt{\left|\frac{2 \partial_{\Delta} g_{\alpha}}{X_{c}^{4} \partial_{X X} g_{\alpha}}\right|} .
$$

The symbol \pm in Eq. (20) accounts for the stable branches $(+)$ and the unstable branches $(-)$ in the reported diagrams (e.g., see Fig. 3). This result, which yields a critical exponent of $1 / 2$ near the existence threshold, is in line with the Landau theory and classical saddlenode bifurcation diagrams. ${ }^{10,29}$ It is important to note that the quantity $J$ that is evaluated at the threshold is only a prefactor that depends on $X_{c}$ and $\alpha$ :

$J=\sqrt{2}\left\{X_{c}^{4}\left[\left(X_{c}^{2}-1\right)^{-3 / 2}+\alpha^{3}\left(\alpha^{2} X_{c}^{2}-1\right)^{-3 / 2}\right]\right\}^{-1 / 2}$

In other words, the exponent of the power-law in Eq. (20) is universal for all minimal surfaces studied here, the prefactor being a function of the considered surface. Figure 17 shows the variations of $\delta a / R$ with $\Delta_{c}-\Delta$ near the threshold for all surfaces.

\section{CONCLUSION}

We have described both theoretically and experimentally the existence and stability of minimal surfaces of revolution materialized by soap films. Our investigations point out the significant role played by the boundary conditions on the domains of existence of these fluid structures. We take advantage of axisymmetry to obtain a set of two equations [Eqs. (17)] whose solutions predict the existence thresholds for any studied configuration. We have also illustrated that soap films can exhibit an hysteretic response due to metastability in these systems. A phenomenological two-step mechanism is introduced to account for the fact that the system is then trapped in a local minimum of energy. ${ }^{13}$ Finally, we have shown that all studied minimal surfaces in the article exhibit a universal behavior near their existence threshold.

\section{ACKNOWLEDGMENTS}

We thank B. Alstatt for preliminary experiments related to those presented in Sec. II C.

\section{Appendix: THE PROFILE OF A CATENOID}

Here, we provide the standard demonstration of Eq. (1), which gives the profile of a catenoid made of a soap film at equilibrium. Indeed, because of symmetry this profile is entirely described by the function $\rho(z)$. The surface energy of the soap film reads

$$
E=2 \gamma \Sigma=2 \gamma 2 \pi \int_{\Sigma} \rho(z) \mathrm{d} s,
$$

where $\gamma$ denotes the surface tension and the factor 2 accounts for the presence of two air-liquid interfaces. We write $\mathrm{d} s=\sqrt{\mathrm{d} \rho^{2}+\mathrm{d} z^{2}}=\sqrt{1+\rho^{\prime 2}(z)} \mathrm{d} z$ with 


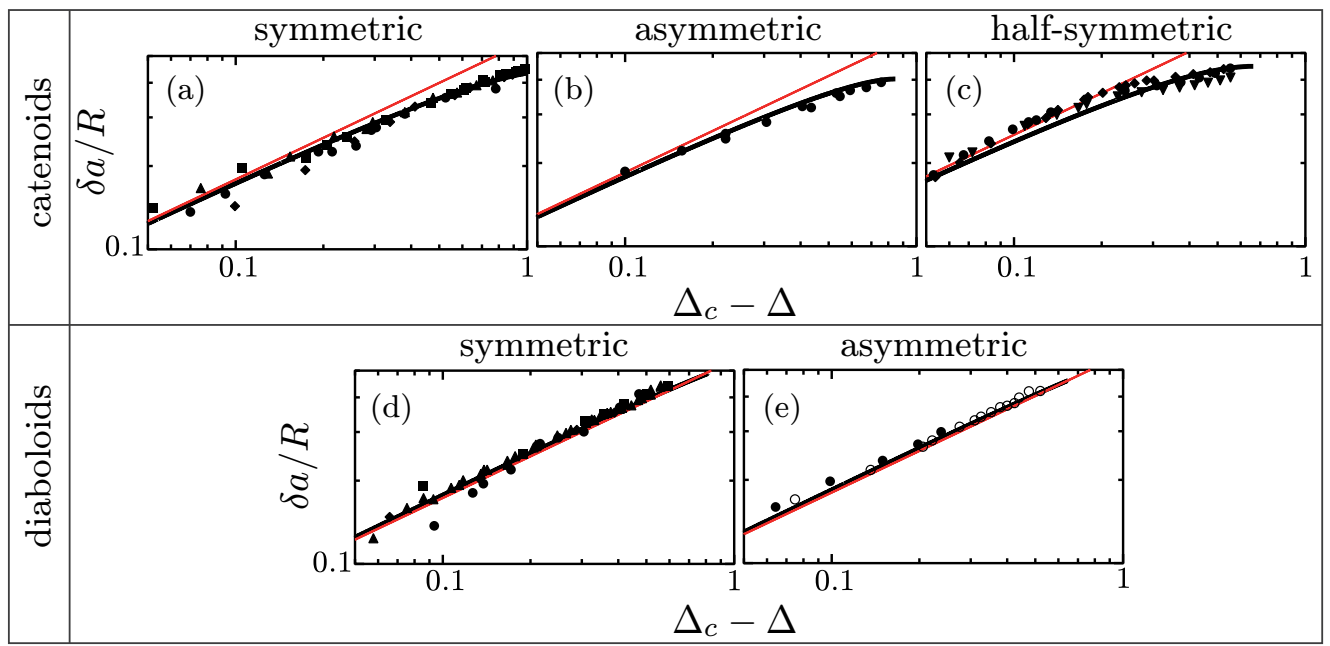

FIG. 17. (Color online) Evolution of $\delta a / R$ with $\Delta_{c}-\Delta$ in the vicinity of the threshold for: (a) symmetric catenoids, (b) an asymmetric catenoid with $\alpha=1.2$, (c) half-symmetric catenoids, (d) symmetric diaboloids, and (e) an asymmetric diaboloid with $\alpha=1.2$. The black and gray (red online) curves correspond, respectively, to the numerical resolution of the boundary conditions and the second-order Taylor series approximation ( $R=R_{1}$ for asymmetric cases).

$\rho^{\prime}(z)=\mathrm{d} \rho / \mathrm{d} z$ in Eq. (A.1) and then minimize this surface energy. By virtue of the variational principle, the Lagrangian of the system $\mathcal{L}=\rho(z) \sqrt{1+\rho^{\prime 2}(z)}$ will satisfy the Euler-Lagrange equation ${ }^{30}$

$$
\frac{\partial \mathcal{L}}{\partial \rho}=\frac{\mathrm{d}}{\mathrm{d} z}\left(\frac{\partial \mathcal{L}}{\partial \rho^{\prime}}\right)
$$

Inspired by analytical mechanics, we define the Hamil- tonian $\mathcal{H}$ as a Legendre transform of $\mathcal{L}$ :

$$
\begin{aligned}
\mathcal{H} & =\frac{\partial \mathcal{L}}{\partial \rho^{\prime}} \rho^{\prime}-\mathcal{L} \\
& =\frac{\rho \rho^{\prime 2}}{\sqrt{1+\rho^{\prime 2}}}-\rho \sqrt{1+\rho^{\prime 2}} \\
& =\frac{-\rho}{\sqrt{1+\rho^{\prime 2}}} .
\end{aligned}
$$

then yields

$$
\frac{\mathrm{d} \rho}{\sqrt{(\rho / a)^{2}-1}}=\mathrm{d} z,
$$

and integrating yields the profile of the catenoid at equilibrium [Eq. (1)]:

$$
\rho(z)=a \cosh \left(\frac{z}{a}+C\right) .
$$

* pascal.panizza@univ-rennes1.fr

$\dagger$ laurent.courbin@univ-rennes1.fr

1 J. Plateau, Statique expérimentale et théorique des liquides soumis aux seules forces moléculaires' (Gauthier-Villars, 1873).

2 C. Isenberg, The Science of soap films and soap bubbles (Dover Publications, 1992).

3 P. Dutta, S. P. Khastgir, and A. Roy, "Steiner trees and spanning trees in six-pin soap films," Am. J. Phys. 78 (2), 215-221 (2010).

4 P. M. Chaikin and T. C. Lubensky, Principles of condensed matter physics (Cambridge University Press, 1995).

${ }^{5}$ F. Otto and B. Rasch, Finding Form: Towards an Archi- tecture of the Minimal (Axel Menges, 1996).

${ }^{6}$ L. Spuybroek, Nox (Thames \& Hudson, 2004).

7 J. Seiwert, M. Monloubou, B. Dollet, and I. Cantat, "Extension of a Suspended Soap Film: A Homogeneous Dilatation Followed by New Film Extraction," Phys. Rev. Lett. 111 (3), 094501-1-5 (2013).

8 D. T. Thi and A. T. Fomenko, Minimal Surfaces, Stratified Multivarifolds, and the Plateau Problem (American Mathematical Society, 1991).

9 L. Hauswirth and F. Pacard, "Higher genus Riemann minimal surfaces," Invent. Math. 169, 569-620 (2007).

10 C. Misbah, Dynamiques complexes et morphogenèse : Introduction aux sciences non linéaires (Springer-Verlag, 
2011).

11 D. R. Lovett and J. Tilley, "Illustrating phase transitions with soap films," Am. J. Phys. 59 (5), 415-421 (1991).

12 L. Durand, "Stability and oscillations of a soap film: an analytic treatment," Am. J. Phys. 49 (4), 334-343 (1981).

13 N. Vandewalle, M. Noirhomme, J. Schockmel, E. Mersch, G. Lumay, D. Terwagne, and S. Dorbolo, "Hysteretic behavior in three-dimensional soap-film rearrangements," Phys. Rev. E 83 (2), 021403-1-5 (2011).

14 M. Ito and T. Sato, "In situ observation of a soap-film catenoid-a simple educational physics experiment," Eur. J. Phys. 31 (2), 357-365 (2010).

15 S. A. Cryer and P. H. Steen, "Collapse of the soap-film bridge: quasistatic description," J. Colloid Interf. Sci. 154 (1), 276-288 (1992).

16 Y. J. Chen and P. H. Steen, "Dynamics of inviscid capillary breakup: collapse and pinchoff of a film bridge," J. Fluid Mech. 341, 245-267 (1997).

17 N. D. Robinson and P. H. Steen, "Observations of singularity formation during the capillary collapse and bubble pinch-off of a soap film bridge," J. Colloid Interf. Sci. 241 (2), 448-458 (2001).

18 F. Müller and R. Stannarius, "Collapse of catenoid-shaped smectic film," Europhys. Lett. 76 (6), 1102-1108 (2006).

19 A. Le Goff, L. Courbin, H. A. Stone, and D. Quéré, "Energy absorption in a bamboo foam," EPL 84 (3), 36001-1-5 (2008).

${ }^{20}$ L. Euler, Opera Omnia (Orell Füssli, 1952).
${ }^{21}$ H. Bouasse, Capillarité - Phénomènes superficiels (Librairie Delagrave, 1924).

${ }^{22}$ Investigations of the formation of these interfacial bubbles and the evolution of their size with the parameters at play will be published elsewhere.

23 P.-G. de Gennes, F. Brochard-Wyart, and D. Quéré, Capillarity and Wetting Phenomena: Drops, Bubbles, Pearls, Waves (Springer, New York, 2004).

24 More information on a diabolo can be found at, e.g., Wikipedia; 〈http://en.wikipedia.org/wiki/Diabolo>.

25 J.-C. Géminard, A. Zywocinski, F. Caillier, and P. Oswald, "Observation of negative line tension from Plateau border regions in dry foam films," Phil. Mag. Lett. 84 (3), 199-204 (2004).

26 S. Besson and G. Debrégeas, "Statics and dynamics of adhesion between two soap bubbles," Eur. Phys. J. E 24 (2), 109-117 (2007).

27 A.-L. Biance, A. Delbos, and O. Pitois, "How topological rearrangements and liquid fraction control liquid foam stability," Phys. Rev. Lett. 106, 068301-1-4 (2011).

28 D. Weaire and S. Hutzler, The Physics of Foams (Oxford University Press, 2000).

29 L. D. Landau and E. M. Lifshitz, Statistical Physics (Pergamon, 1980).

30 J.-L. Lagrange, Mécanique analytique (Gauthier-Villars, 1889). 